\title{
QuantifQuantile: An R Package for Performing Quantile Regression Through Optimal Quantization
}

by Isabelle Charlier, Davy Paindaveine and Jérôme Saracco

\begin{abstract}
In quantile regression, various quantiles of a response variable $Y$ are modelled as functions of covariates (rather than its mean). An important application is the construction of reference curves/surfaces and conditional prediction intervals for $Y$. Recently, a nonparametric quantile regression method based on the concept of optimal quantization was proposed. This method competes very well with $k$-nearest neighbor, kernel, and spline methods. In this paper, we describe an R package, called QuantifQuantile, that allows to perform quantization-based quantile regression. We describe the various functions of the package and provide examples.
\end{abstract}

\section{Introduction}

In numerous applications, quantile regression is used to evaluate the impact of a $d$-dimensional covariate $X$ on a (scalar) response variable $Y$. Quantile regression is an interesting alternative to standard regression whenever the conditional mean does not provide a satisfactory picture of the conditional distribution. Denoting by $F(\cdot \mid x)$ the conditional distribution of $Y$ given $X=x$, the conditional quantile functions

$$
x \mapsto q_{\alpha}(x)=\inf \{y \in \mathbb{R}: F(y \mid x) \geq \alpha\}, \quad \alpha \in(0,1),
$$

indeed always yield a complete description of the conditional distribution. For our purposes, it is useful to recall that the conditional quantiles in (1) can be equivalently defined as

$$
q_{\alpha}(x)=\arg \min _{a \in \mathbb{R}} \mathrm{E}\left[\rho_{\alpha}(Y-a) \mid X=x\right],
$$

where $\rho_{\alpha}(z)=\alpha z \mathbb{I}_{[z \geq 0]}-(1-\alpha) z \mathbb{I}_{[z<0]}$ is the so-called check function.

For fixed $\alpha$, the quantile functions $x \mapsto q_{\alpha}(x)$ provide reference curves (when $d=1$ ), one for each value of $\alpha$. For fixed $x$, they provide conditional prediction intervals of the form $I_{\alpha}=\left[q_{\alpha}(x), q_{1-\alpha}(x)\right]$ $(\alpha<1 / 2)$. Such reference curves and prediction intervals are widely used, e.g. in economics, ecology, or lifetime analysis. In medicine, they are used to provide reference growth curves for children's height and weight given their age.

Many approaches have been developed to estimate conditional quantiles. After the seminal paper of Koenker and Bassett (1978) that introduced linear quantile regression, much effort has been made to consider nonparametric quantile regression. The most classical procedures in this vein are the nearest neighbor estimators (Bhattacharya and Gangopadhyay, 1990), the (kernel) local linear estimators (Yu and Jones, 1998) or the spline-based estimators (Koenker et al., 1994; Koenker and Mizera, 2004). For related work, we also refer to, e.g. Fan et al. (1994), Gannoun et al. (2002), Muggeo et al. (2013) and Yu et al. (2003). There also exists a wide variety of $\mathrm{R}$ functions/packages dedicated to the estimation of conditional quantiles. Among them, let us cite the functions rqss (only for $d \leq 2$ ) and gcrq (only for $d=1$ ) from the packages quantreg (Koenker, 2015) and quantregGrowth (Muggeo, 2015), respectively.

Recently, Charlier et al. (2015a) proposed a nonparametric quantile regression method based on the concept of optimal quantization. Optimal quantization replaces the (typically continuous) covariate $X$ with a discretized version $\widetilde{X}^{N}$ obtained by projecting $X$ on a collection of $N$ points (these $N$ points, that form the quantization grid, are chosen to minimize the $\mathrm{L}_{p}$-norm of $X-\widetilde{X}^{N}$; see below). As shown in Charlier et al. (2015b), the resulting conditional quantile estimators compete very well with their classical competitors.

The goal of this paper is to describe an R package, called QuantifQuantile (Charlier et al., 2015c), that allows to perform quantization-based quantile regression. This includes the data-driven selection of the grid size $N$ (that plays the role of a tuning parameter), the construction of the corresponding quantization grid, the computation of the resulting sample conditional quantiles, as well as (for $d \leq 2$ ) their graphical representation.

The paper is organized as follows. The first section briefly recalls the construction of quantizationbased quantile regression introduced in Charlier et al. $(2015 a, b)$ and explains the various steps needed to obtain the resulting estimators. The second section lists the main functions of QuantifQuantile and 
describes their inputs and outputs. Finally, the third section provides several examples that illustrate the use of the various functions. We conclude the paper by comparing our method with $\mathrm{R}$ alternatives on a real data set. An illustration of the function computing optimal quantization grids is given in the Appendix, which can be of independent interest in numerical probability, finance or numerical integration where quantization is extensively used (Pagès, 1998; Pagès et al., 2004).

\section{Quantile regression through quantization}

As mentioned above, the R package we describe in this paper implements the Charlier et al. $(2015 \mathrm{a}, \mathrm{b})$ quantization-based methodology to perform nonparametric quantile regression. This section describes this methodology.

\section{Approximating population conditional quantiles through quantization}

Let $\gamma^{N} \in\left(\mathbb{R}^{d}\right)^{N}$ be a grid of size $N$, that is, a collection of $N$ points in $\mathbb{R}^{d}$. For any $x \in \mathbb{R}^{d}$, we will denote by $\tilde{x}^{N}=\operatorname{Proj}_{\gamma^{N}}(x)$ the projection of $x$ onto this grid, that is, the point of $\gamma^{N}$ that is closest to $x$ (absolute continuity assumption makes ties unimportant in the sequel). This allows to approximate the $d$-dimensional covariate $X$ by its quantized version $\widetilde{X} \gamma^{N}$.

Obviously, the choice of the grid has a significant impact on the quality of this approximation. Under the assumption that $\|X\|_{p}:=\mathrm{E}\left[|X|^{p}\right]^{1{ }_{p}^{p}}<\infty$ (throughout, $|\cdot|$ denotes the Euclidean norm), optimal quantization selects the grid $\gamma^{N}$ that minimizes the $\mathrm{L}_{p}$-quantization error $\left\|X-\tilde{X}^{\gamma^{N}}\right\|_{p}$. Such an optimal grid exists under the assumption that the distribution $P_{X}$ of $X$ does not charge any hyperplane, i.e. under the assumption that $P_{X}[H]=0$ for any hyperplane $H$ (Pagès, 1998). In practice, an optimal grid is constructed using a stochastic gradient algorithm (see the following section). For more details on quantization, the reader may refer to Pagès (1998) and Graf and Luschgy (2000).

Based on optimal quantization of $X$, we can approximate the conditional quantile $q_{\alpha}(x)$ in (2) by

$$
\widetilde{q}_{\alpha}^{N}(x):=\arg \min _{a \in \mathbb{R}} \mathrm{E}\left[\rho_{\alpha}(Y-a) \mid \widetilde{X}^{N}=\tilde{x}^{N}\right],
$$

where $\widetilde{X}^{N}$ (resp., $\tilde{x}^{N}$ ) denotes the projection of $X$ (resp., $x$ ) onto an optimal grid. It is shown in Charlier et al. (2015a) that under mild assumptions, ${ }^{1} \widetilde{q}_{\alpha}^{N}(x)$ converges to $q_{\alpha}(x)$ as $N \rightarrow \infty$, uniformly in $x$.

\section{Obtaining an optimal $N$-grid}

As we will see below, whenever independent copies $\left(X_{1}^{\prime}, Y_{1}\right)^{\prime}, \ldots,\left(X_{n}^{\prime}, Y_{n}\right)^{\prime}$ of $\left(X^{\prime}, Y\right)^{\prime}$ are available, the first step to obtain a sample version of (3) is to compute an optimal $N$-grid (we assume here that $N$ is fixed). As already mentioned, this can be done through a stochastic gradient algorithm. This algorithm, called Competitive Learning Vector Quantization (CLVQ) when $p=2$, is an iterative procedure that operates as follows :

- First, an initial grid $-\hat{\gamma}^{N, 0}$, say - is chosen by sampling randomly without replacement among the $X_{i}^{\prime}$ s.

- Second, $n$ iterations are performed (one for each observation available). The grid $\hat{\gamma}^{N, t}=$ $\left(\hat{\gamma}_{1}^{N, t}, \ldots, \hat{\gamma}_{N}^{N, t}\right)$ at step $t$ is obtained through

$$
\hat{\gamma}_{i}^{N, t}= \begin{cases}\hat{\gamma}_{i}^{N, t-1}-\delta_{t}\left|\hat{\gamma}_{i}^{N, t-1}-X_{t}\right|^{p-1} \frac{\hat{\gamma}_{i}^{N, t-1}-X_{t}}{\left|\hat{\gamma}_{i}^{N, t-1}-X_{t}\right|} & \text { if } \operatorname{Proj}_{\hat{\gamma}^{N, t-1}}\left(X_{t}\right)=\hat{\gamma}_{i}^{N, t-1}, \\ \hat{\gamma}_{i}^{N, t-1} & \text { otherwise, }\end{cases}
$$

where $\left(\delta_{t}\right), t \in \mathbb{N}_{0}$, is a deterministic sequence in $(0,1)$ such that $\sum_{t} \delta_{t}=\infty$ and $\sum_{t} \delta_{t}^{2}<\infty$. At the $t^{\text {th }}$ iteration, only one point of the grid moves, namely the one that is closest to $X_{t}$.

The optimal grid provided by this algorithm is then $\hat{\gamma}^{N, n}$.

\section{Estimating conditional quantiles}

Assume now that a sample $\left(X_{1}^{\prime}, Y_{1}\right)^{\prime}, \ldots,\left(X_{n}^{\prime}, Y_{n}\right)^{\prime}$ as above is indeed available. The sample analog of (3) is then defined as follows :

\footnotetext{
${ }^{1}$ Our method actually requires assumptions on the link function between $Y$ and $X$ and on the error term of the model. These assumptions in particular guarantee that, for any $x$, the conditional distribution of $Y$ given $X=x$ is absolutely continuous; we refer to Charlier et al. (2015a) for details.
} 
(S1) First, we compute the optimal grid $\hat{\gamma}^{N, n}$ through the stochastic gradient algorithm just described, and we write $\widehat{X}_{i}^{N}=\operatorname{Proj}_{\hat{\gamma}^{N, n}}\left(X_{i}\right), i=1, \ldots, n$.

(S2) Then, the conditional quantiles are estimated by

$$
\widehat{q}_{\alpha}^{N, n}(x)=\arg \min _{a \in \mathbb{R}} \sum_{i=1}^{n} \rho_{\alpha}\left(Y_{i}-a\right) \mathbb{I}_{\left[\widehat{X}_{i}^{N}=\hat{x}^{N}\right]},
$$

where $\hat{x}^{N}=\operatorname{Proj}_{\hat{\gamma}^{N, n}}(x)$. In practice, $\widehat{q}_{\alpha}^{N, n}(x)$ is simply evaluated as the sample $\alpha$-quantile of the $Y_{i}^{\prime}$ s for which $\widehat{X}_{i}^{N}=\hat{x}^{N}$.

It is shown in Charlier et al. (2015a) that under mild assumptions, $\widehat{q}_{\alpha}^{N, n}(x)$, for any fixed $N$ and $x$, converges in probability to $\widetilde{q}_{\alpha}^{N}(x)$ as $n \rightarrow \infty$, provided that quantization is based on $p=2$.

When the sample size $n$ is small to moderate ( $n \leq 300$, say), the estimated reference curves $x \mapsto$ $\widehat{q}_{\alpha}^{N, n}(x)$ typically are not smooth. To improve on this, Charlier et al. $(2015 \mathrm{a}, \mathrm{b})$ introduced the following bootstrapped version of the estimator in (4). For some positive integer $B$, generate $B$ samples of size $n$ from the original sample $\left\{\left(X_{i}^{\prime}, Y_{i}\right)^{\prime}\right\}_{i=1, \ldots, n}$ with replacement. From each of these bootstrap samples, the stochastic gradient algorithm provides an "optimal" grid, using these bootstrapped samples to perform the iterations. The bootstrapped estimator of conditional quantile is then

$$
\bar{q}_{\alpha, B}^{N, n}(x)=\frac{1}{B} \sum_{b=1}^{B} \hat{q}_{\alpha}^{(b)}(x)
$$

where $\hat{q}_{\alpha}^{(b)}(x)=\hat{q}_{\alpha}^{(b), N, n}(x)$ denotes the estimator in (4) computed on the basis of the $b^{\text {th }}$ optimal grid. We stress that, when computing $\hat{q}_{\alpha}^{(b)}(x)$, the original sample is used in (S2); the bootstrapped samples are only used to provide the $B$ different grids. As shown in Charlier et al. (2015a,b), the bootstrapped reference curves are much smoother than the original ones. Of course, $B$ should be chosen large enough to make the bootstrap useful, but also small enough to keep the computational burden under control. For $d=1$, we usually choose $B=50$.

\section{Selecting the grid size $N$}

Both for the original estimators $\widehat{q}_{\alpha}^{N, n}(x)$ and for their bootstrapped versions $\bar{q}_{\alpha, B}^{N, n}(x)$, an appropriate value of $N$ should be identified. If $N$ is too small, then reference curves will have a large bias, while if $N$ is too large, then they will show much variability. This leads to the usual bias/variance trade-off that is to be achieved when selecting the value of a smoothing parameter in nonparametric statistics.

Charlier et al. (2015b) proposed the following data-driven method to choose $N$. Let $\left\{x_{1}, \ldots, x_{J}\right\}$ be a set of $x$-values at which we want to estimate $q_{\alpha}(x)$ (the $x_{j}$ 's are for instance chosen equispaced on the support of $X$ ) and let $\mathcal{N}$ be a finite collection of $N$-values (this represents the values of $N$ one allows for and should typically be chosen according to the sample size $n$ ). Ideally, we would like to select the optimal value of $N$ as

$$
N_{\alpha ; \mathrm{opt}}^{-}=\arg \min _{N \in \mathcal{N}} \operatorname{ISE}_{\alpha}^{-}(N), \quad \text { with } \operatorname{ISE}_{\alpha}^{-}(N)=\frac{1}{J} \sum_{j=1}^{J}\left(\bar{q}_{\alpha, B}^{N, n}\left(x_{j}\right)-q_{\alpha}\left(x_{j}\right)\right)^{2} .
$$

This, however, is infeasible, since the population quantiles $q_{\alpha}\left(x_{j}\right)$ are unknown. This is why we draw $\widetilde{B}$ extra bootstrap samples (still of size $n$ ) from the original sample and consider

$$
\hat{N}_{\alpha ; \mathrm{opt}}^{-}=\arg \min _{N \in \mathcal{N}} \widehat{\operatorname{ISE}}_{\alpha}^{-}(N), \quad \text { with } \widehat{\operatorname{ISE}}_{\alpha}^{-}(N)=\frac{1}{J} \sum_{j=1}^{J}\left(\frac{1}{\widetilde{B}} \sum_{\tilde{b}=1}^{\widetilde{B}}\left(\bar{q}_{\alpha, B}^{N, n}\left(x_{j}\right)-\hat{q}_{\alpha}^{(\tilde{b})}\left(x_{j}\right)\right)^{2}\right),
$$

where $\hat{q}_{\alpha}^{(\tilde{b})}\left(x_{j}\right)$, for $\tilde{b}=1 \ldots, \tilde{B}$, makes use of this $\tilde{b}^{\text {th }}$ new bootstrap sample; more precisely, the bootstrap sample is still only used to perform the iterations of the algorithm, whereas the original sample is used in both the initial grid and in (S2).

As shown in Charlier et al. (2015b) through simulations, both $N \mapsto \operatorname{ISE}_{\alpha}^{-}(N)$ and $N \mapsto \widehat{\operatorname{ISE}}_{\alpha}^{-}(N)$ are essentially convex in $N$ and lead to roughly the same minimizers. This therefore provides a feasible way to select a reasonable value of $N$ for the estimator $\bar{q}_{\alpha, B}^{N, n}(x)$ in (5). Note that this also applies to $\hat{q}_{\alpha}^{N, n}(x)$ by simply taking $B=1$ in the procedure above.

If quantiles are to be estimated at various orders $\alpha$, (7) will provide an optimal $N$-value for each $\alpha$. It may then happen, in principle, that the resulting reference curves cross, which is of course undesirable. 
One way to guarantee that no such crossings occur is to identify a common $N$-value for the various $\alpha^{\prime}$ s. In such a case, $N$ will be chosen as

$$
\hat{N}_{\text {opt }}^{-}=\arg \min _{N \in \mathcal{N}} \widehat{\operatorname{ISE}}^{-}(N), \quad \text { with } \widehat{\operatorname{ISE}}^{-}(N)=\sum_{\alpha} \widehat{\operatorname{ISE}}_{\alpha}^{-}(N),
$$

where the sum is computed over the various $\alpha$-values considered.

Charlier et al. (2015b) performed extensive comparisons between the quantization-based estimators in (5) - based on the efficient data-driven selection method for $N$ just described - and some of their main competitors, namely estimators obtained from spline, $k$-nearest neighbor, and kernel methods. This revealed that the quantization-based estimators compete well in all cases, and often dominates their competitors (in terms of integrated square errors), particularly so for complex link functions; see Charlier et al. (2015b) for details.

Unlike the local linear and local constant estimators from Yu and Jones (1998), that are usually based on a global-in- $x$ bandwidth, our quantization-based estimators are locally adaptive in the sense that, when estimating $q_{\alpha}(x)$, the "working bandwidth" - that is, the size of the quantization cell containing $x$-depends on $x$. The $k$-nearest neighbor $(k \mathrm{NN})$ estimator is closer in spirit to quantizationbased estimators but always selects $k$ neighbors, irrespective of the $x$-value considered, whereas the number of $X_{i}$ 's in the quantization cell of $x$ may depend on $x$. This subtle local-in- $x$ behavior may explain the good empirical performances of quantization-based estimators over kernel and nearestneighbor competitors. Finally, spline methods (implemented in R with the rqss and qss functions) tend to perform poorly for complex link functions, since they always provide piecewise linear reference curves (Koenker et al., 1994). Moreover, the current implementation of the rqss function only supports dimensions 1 and 2, whereas our package allows to compute quantization-based estimators in any dimension $d$.

\section{The QuantifQuantile package}

This section provides a description of the various functions offered in the R package QuantifQuantile. We first detail the three functions that allow to estimate conditional quantiles through quantization. Then we describe a function computing optimal quantization grids.

\section{Conditional quantile estimation}

QuantifQuantile is composed of three main functions that each provide estimated conditional quantiles in (4)-(5). These functions work in a similar way but address different values of $d$ (recall that $d$ is the dimension of the covariate vector $X)$ :

- The function QuantifQuantile is suitable for $d=1$.

- The function QuantifQuantile.d2 addresses the case $d=2$.

- Finally, Quanti fQuantile.d can deal with an arbitrary value of $d$.

Combined with the plot function, the first two functions provide reference curves and reference surfaces, respectively. No graphical outputs can be obtained from the third function if $d>2$.

The three functions share the same arguments, but not necessarily the same default values. For each function, using args() displays the various arguments and corresponding default values :

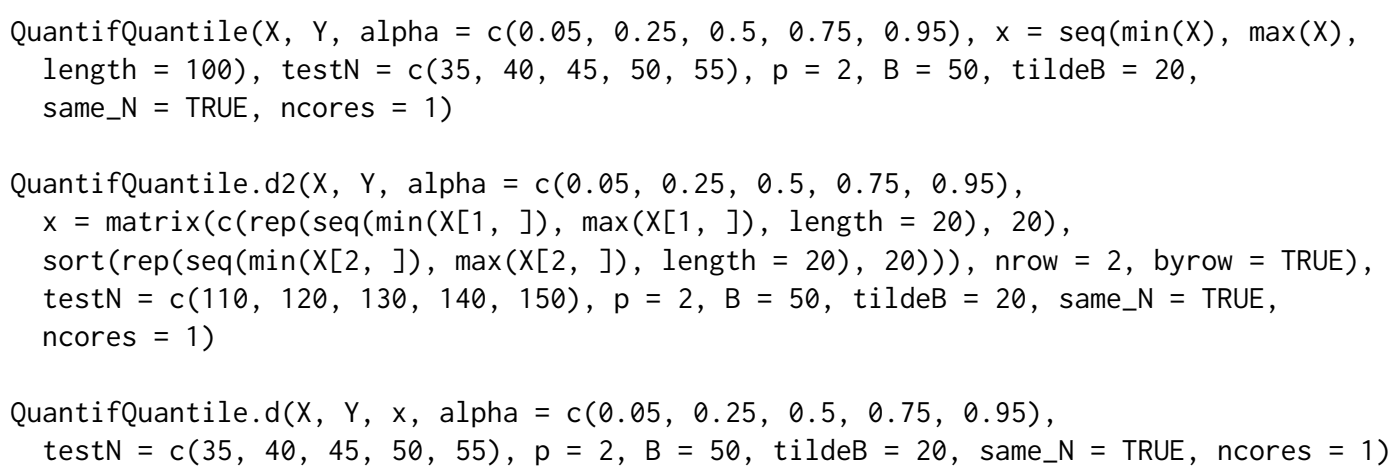

We now give more details on these arguments.

- $\mathrm{X}$ : a $d \times n$ real array (required by all three functions, a vector of length $n$ for Quanti fQuantile). The columns of this matrix are the $X_{i}{ }^{\prime} \mathrm{s}, i=1, \ldots, n$. 
- Y: an $n \times 1$ real array (required by all three functions). This vector collects the $Y_{i}{ }^{\prime} \mathrm{s}, i=1, \ldots, n$.

- alpha: an $r \times 1$ array with components in $(0,1)$ (optional for all three functions). This vector contains the orders for which $q_{\alpha}(x)$ should be estimated.

- x: a $d \times J$ real array (optional for QuantifQuantile and QuantifQuantile.d2, required by QuantifQuantile.d). The columns of this matrix are the $x_{j}{ }^{\prime}$ s at which the quantiles $q_{\alpha}\left(x_{j}\right)$ are to be estimated. If $x$ is not provided when calling QuantifQuantile, then it is set to a vector of $J=100$ equispaced values between the minimum and the maximum of the $X_{i}$ 's. If this argument is not provided when calling QuantifQuantile.d2, then the default for $x$ is a matrix whose $J=20^{2}=400$ column vectors are obtained as follows: 20 equispaced values are considered between the minimum and maximum values of the $\left(X_{i}\right)_{1}$ 's and similarly for the second component. The 400 column vectors of the default $x$ are obtained by considering all combinations of those 20 values for the first component with the 20 values for the second one ${ }^{2}$.

- testN: an $m \times 1$ vector of pairwise distinct positive integers (optional for all three functions). The entries of this vector are the elements of the set $\mathcal{N}$ in (7)-(8), hence are the $N$-values for which the $\widehat{\mathrm{ISE}}_{\alpha}^{-}$quantity considered will be evaluated. The default is $(35,40, \ldots, 55)$ but it is strongly recommended to adapt it according to the sample size $n$ at hand.

- $p$ : a real number larger than or equal to one (optional for all three functions). This is the parameter $p$ to be used when performing optimal quantization in $\mathrm{L}_{p}$-norm.

- B: a positive integer (optional for all three functions). This is the number of bootstrap replications $B$ to be used in (5).

- tildeB: a positive integer (optional for all three functions). This is the number of bootstrap replications $\widetilde{B}$ to be used when determining $\hat{N}_{\alpha ; \text { opt }}^{-}$or $\hat{N}_{\text {opt }}^{-}$.

- same_N: a boolean variable (optional for all three functions). If same_N=TRUE, then a common value of $N$ (that is, $\hat{N}_{\text {opt }}^{-}$in (8)) will be selected for all $\alpha$ 's. If same_N=FALSE, then optimal values of $N$ will be chosen independently for the various of $\alpha$ (which will provide several $\hat{N}_{\alpha ; \text { opt }}^{-}$, as in (7)).

- ncores: number of cores to use. These functions can use parallel computation to save time by increasing this parameter. Parallel computation relies on mclapply from parallel package, hence is not available on Windows.

All three functions return the following list of objects, which is of class 'Quant i fQuantile' :

- hatq_opt: an $r \times J$ real array (where $r$ is the number of $\alpha$-values considered). If same_N=TRUE, then the entry $(i, j)$ of this matrix is $\bar{q}_{\alpha_{i}, B}^{\hat{N}_{\text {opt }}^{-}, n}\left(x_{j}\right)$. If same_N=FALSE, then it is rather $\bar{q}_{\alpha_{i}, B}^{\hat{N}_{\alpha_{i} \text { opt }}^{-}, n}\left(x_{j}\right)$. This object can also be returned using the usual fitted. values function.

- N_opt: a positive integer (if same_N=TRUE) or an $r \times 1$ array of positive integers (if same_N=FALSE). Depending on same_N, this provides the value of $\hat{N}_{\text {opt }}^{-}$or the vector $\left(\hat{N}_{\alpha_{1} ; \text { opt }}^{-}, \ldots, \hat{N}_{\alpha_{r} ; \text { opt }}^{-}\right)$.

- hatISE_N: an $r \times m$ real array. The entry $(i, j)$ of this matrix is $\widehat{\operatorname{ISE}}_{\alpha_{i}}\left(N_{j}\right)$. Plotting this for fixed $\alpha$ or plotting its average over the various $\alpha$, in both cases over testN, allows to assess the global convexity of these ISEs. Hence, it can be used to indicate whether or not the choice of testN was adequate. This will be illustrated in the examples below.

- hatq_N: an $r \times J \times m$ real array. The entry $(i, j, \ell)$ of this matrix is $\bar{q}_{\alpha_{\ell}, B}^{N_{\ell}, n}\left(x_{j}\right)$, where $N_{\ell}$ is the $\ell^{\text {th }}$ entry of the argument testN. From this output, it is easy by fixing the third entry to get the matrix of the $\bar{q}_{\alpha_{i}, B}^{N, n}\left(x_{j}\right)$ values for any $N$ in testN.

- The arguments $X, Y, X$, alpha, and testN are also reported in this response list.

Moreover, when the optimal value N_opt selected is on the boundary of testN, which means that testN most likely was not well chosen, a warning message is printed.

The 'QuantifQuantile' class response can be used as argument of the functions plot (only for $d \leq 2)$, summary and print. The plot function draws the observations and plots the estimated conditional quantile curves $(d=1)$ or surfaces $(d=2)$ - for $d=2$, the rgl package is used (Adler et al., 2015), which allows to change the perspective in a dynamic way. In order to illustrate the selection of $N$, the function plot also has an optional argument ise. Setting this argument to TRUE (the default is FALSE), this function, that can be used for any dimension $d$, provides the plot (against $N$ ) of the $\widehat{\mathrm{ISE}}_{\alpha}^{-}$ and $\widehat{\text { ISE }}$ quantities in (7) or in (8), depending on the choice same_N=FALSE or same_N=TRUE, respectively; see the examples below for details. If $d \leq 2$, it also returns the fitted quantile curves or surfaces.

\footnotetext{
${ }^{2}$ Since the number $J$ of points in a default value of $x$ obtained in this fashion would increase exponentially with the dimension $d$, we did not adopt the same approach for $d \geq 3$.
} 


\section{Computing optimal grids}

Besides the functions that allow to estimate conditional quantiles and to plot the corresponding reference curves/surfaces, QuantifQuantile provides a function that computes optimal quantization grids. This function, called choice.grid, admits the following arguments :

- $\mathrm{x}$ : a $d \times n$ real array (required). The columns of this matrix are the $X_{i}{ }^{\prime} \mathrm{s}, i=1, \ldots, n$, for which the optimal quantization grid should be determined. Each point of $X$ is used as a stimulus in the stochastic gradient algorithm to get an optimal grid.

- $\mathrm{N}$ : a positive integer (required). The size of the desired quantization grid.

- ng: a positive integer (optional). The number of desired quantization grids. The default is 1 .

- $p$ : a real number larger than or equal to one (optional). This is the parameter $p$ used in the quantization error. The default is 2 .

In some cases, it may be necessary to have several quantization grids, such as in (5), where B + tildeB grids are needed. The three functions computing quantization-based conditional quantiles then call the function choice.grid with $\mathrm{ng}>1$. In such case, the various grids are obtained using as stimuli a resampling version of $X$ (the $X_{t}$ 's in the previous section).

The output is a list containing the following elements :

- init_grid: a $d \times \mathrm{N} \times \mathrm{ng}$ real array. The entry $(i, j, \ell)$ of this matrix is the $i^{\text {th }}$ component of the $j^{\text {th }}$ point of the $\ell^{\text {th }}$ initial grid.

- opti_grid: a $d \times \mathrm{N} \times \mathrm{ng}$ real array. The entry $(i, j, \ell)$ of this matrix is the $i^{\text {th }}$ component of the $j^{\text {th }}$ point of the $\ell^{\text {th }}$ optimal grid provided by the algorithm.

\section{Illustrations}

In this section, we illustrate on several examples the use of the functions described above. Examples 13 restrict to QuantifQuantile/QuantifQuantile.d2 and provide graphical representations in each case. Example 4 deals with a three-dimensional covariate, without graphical representation. An illustration of the function choice.grid is given in the Appendix.

\section{Example 1: Simulated data with one-dimensional covariate}

We generate a random sample $\left(X_{i}, Y_{i}\right)^{\prime}, i=1, \ldots, n=300$, where the $X_{i}$ 's are uniformly distributed over the interval $(-2,2)$ and where the $Y_{i}^{\prime}$ 's are obtained by adding to $X_{i}^{2}$ a standard normal error term that is independent of $X_{i}$ :

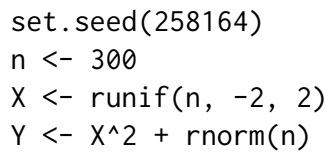

We test the number $N$ of quantizers between 10 and 30 by steps of 5 and we do not change the default values of the other arguments. We then run the function QuantifQuantile with these arguments and stock the response in res.

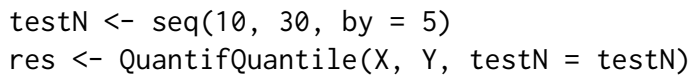

No warning message is printed, which means that this choice of test $\mathrm{N}$ was adequate. To assess this in a graphical way, we use the function plot with ise argument set to TRUE that plots hatISEmean_N (obtained by taking the mean of hatISE_N over alpha) against the various $N$-values in testN.

$\operatorname{plot}($ res, ise $=$ TRUE $)$

Figure 1a provides the resulting graph, which confirms that testN was well chosen since hat ISEmean_N is larger for smaller and larger values of $N$ than N_opt. We then plot the corresponding estimated conditional quantiles curves in Figure 1b. The default colors of the points and of the curves are changed by using the col. plot argument. This argument is a vector of size $1+$ length(alpha), whose first component fixes the color of the data points and whose remaining components determine the colors of the various reference curves.

col.plot <- c("grey", "red", "orange", "green", "orange", "red")

plot (res, col.plot $=$ col.plot, $x l a b=" X ", y l a b=" Y ")$ 


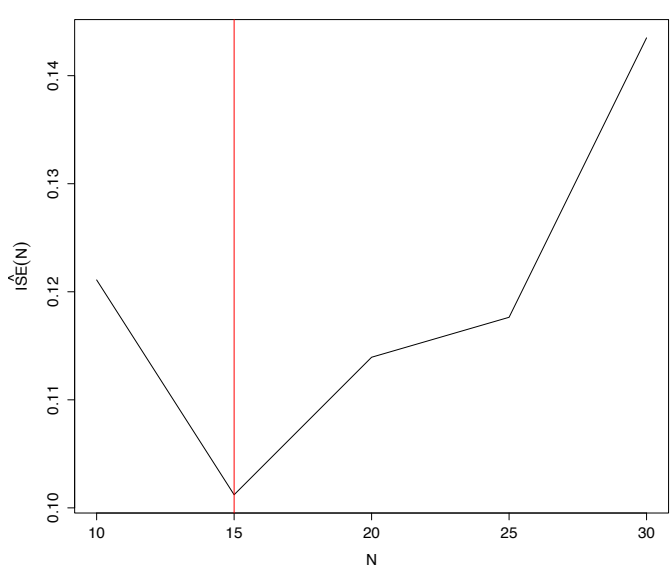

(a)

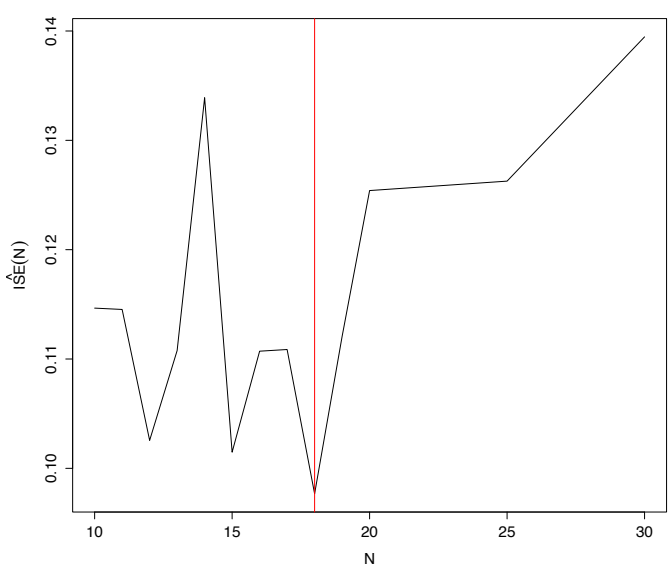

(c)

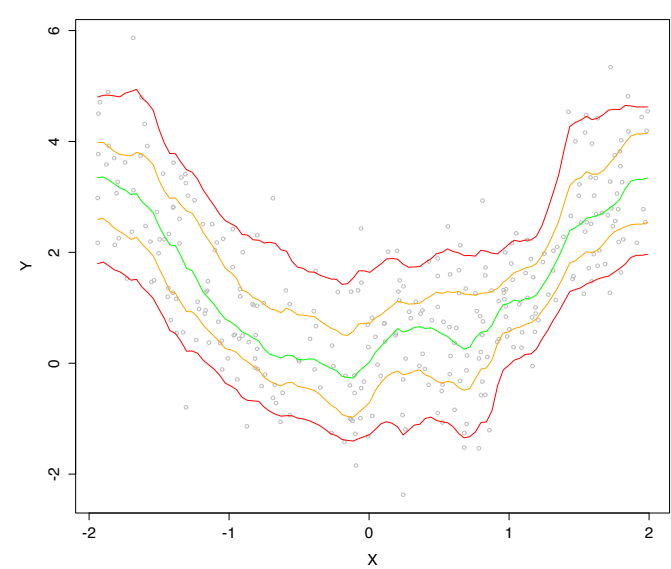

(b)

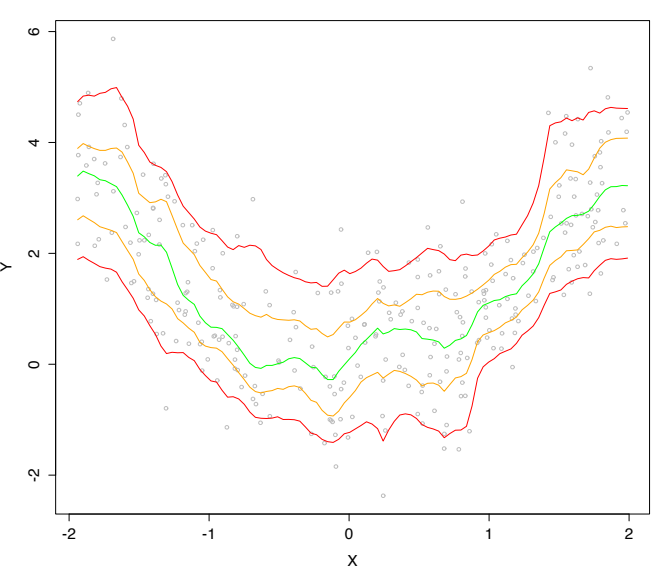

(d)

Figure 1: For the sample considered in Example 1, this figure provides (a) the plot of $N \mapsto \widehat{\mathrm{ISE}}^{-}(N)$ with $N \in\{10,15,20,25,30\}$, and (b) the resulting reference curves. The panels (c)-(d) provide the corresponding plots when taking $N \in\{10,11,12, \ldots, 19,20,25,30\}$.

It is natural to make the grid testN finer. Of course, the more $N$-values we test, the longer it takes. This is why we adopted this two-stage approach, where the goal of the first stage was to get a rough approximation of the optimal $N$-value. In the second stage, we can then refine the grid only in the vicinity of the value $\mathrm{N}_{-}$opt obtained in the first stage.

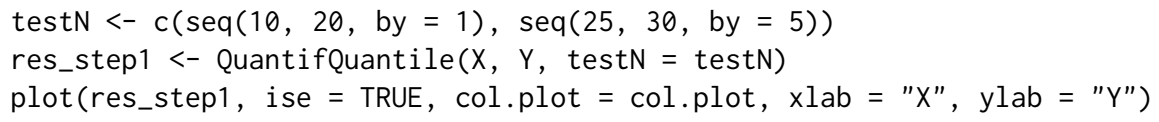

The resulting graphs are provided in Figures $1 c-1 d$, respectively. We observe that the value of N_opt is made more precise, since we now get $N_{\text {_opt }}=18$ instead of 15 . The resulting estimated conditional quantiles curves in Figure $1 \mathrm{~b}$ are very similar to the ones in Figure $1 \mathrm{~d}$.

So far, we used the default value same_N=TRUE, which leads to selecting an $N$-value that is common to all $\alpha$ 's. For the sake of comparison, we now explore the results for same_N=FALSE.

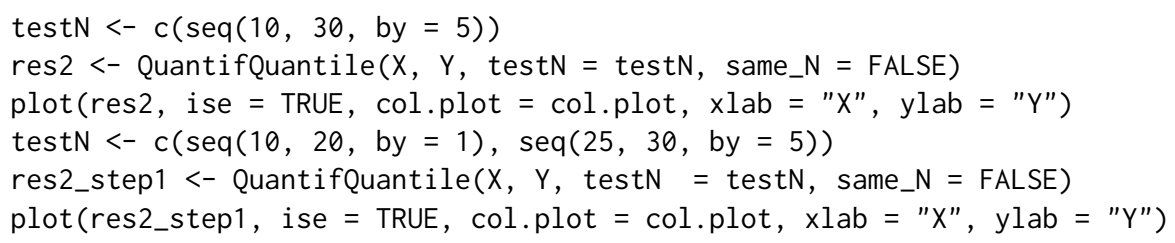

The results are provided in Figure 2. Comparing the left panels of Figures 1 and 2, we see that 


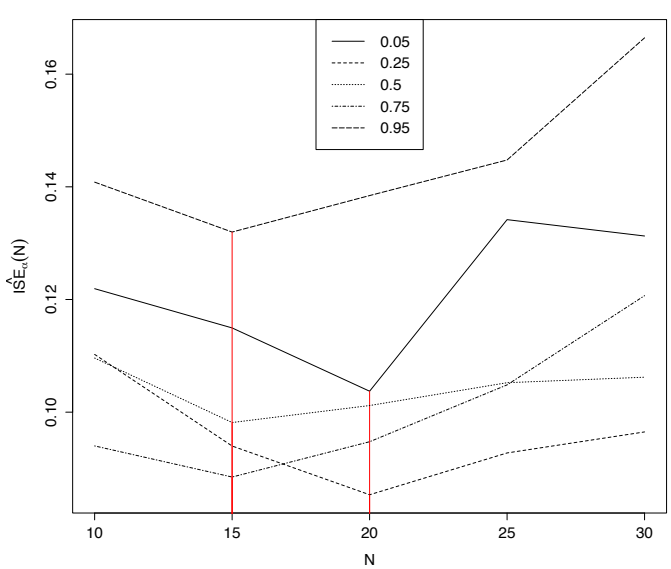

(a)

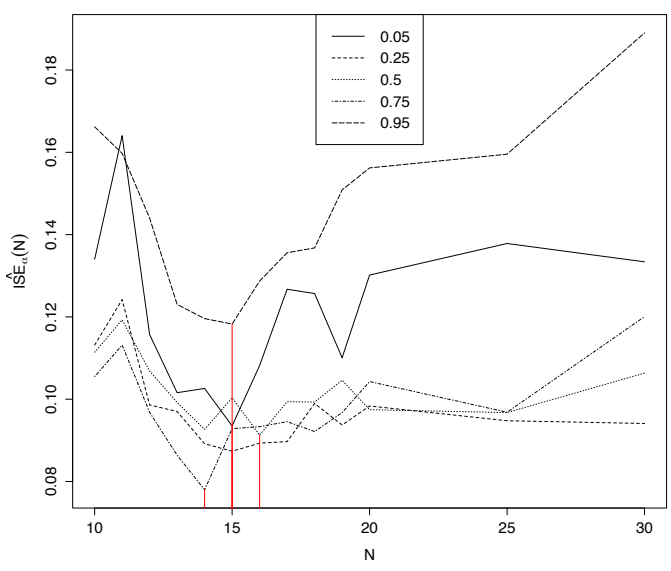

(c)

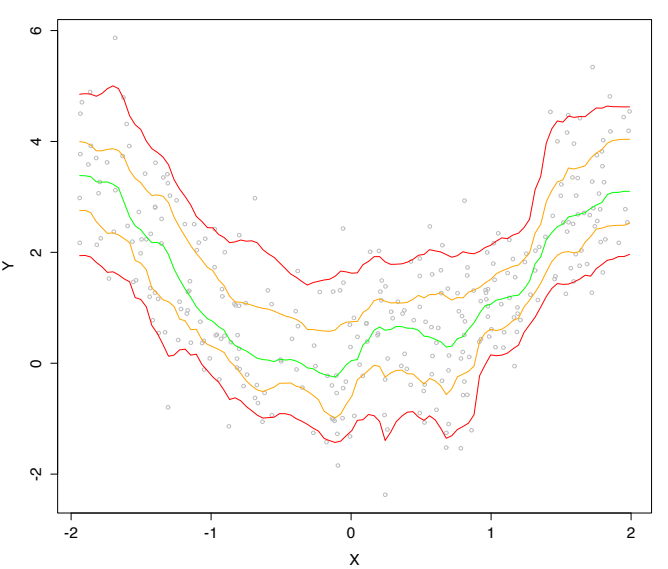

(b)

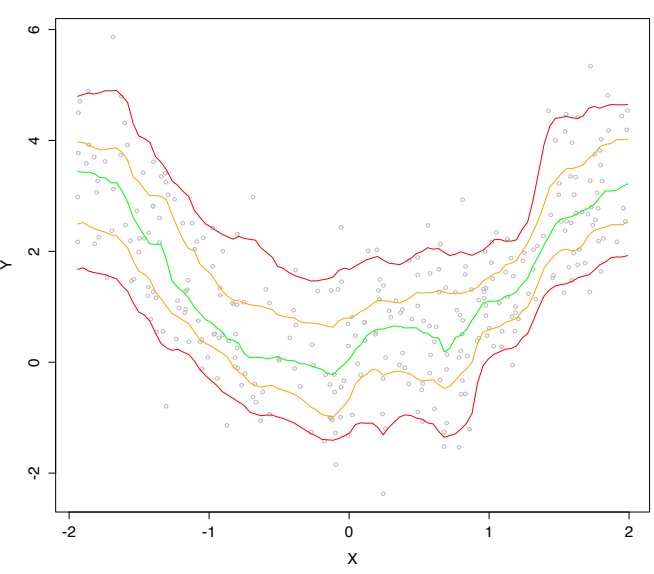

(d)

Figure 2: The same results as in Figure 1, but when selecting optimal values of $N$ separately for each $\alpha$.

when choosing $N$ by steps of five, we find N_opt $=15$ with same_N $=$ TRUE and N_opt $=15$ or 20 (depending on alpha) for same_N = FALSE. When we refine the grid testN, we find analogously N_opt $=18$ for same_N $=$ TRUE and N_opt $=14,15$, or 16 for same_N $=$ FALSE. In the present setup, thus, both methods provide relatively close optimal $N$-values, which explains why the corresponding estimated reference curves are so similar (see the right panels of Figures 1 and 2). Therefore, the grid of $N$-values tested in Figure 1, that may seem too coarse at first sight, actually provides fitted curves that are as satisfactory as those associated with the finer grid in Figure 2.

\section{Example 2: Simulated data with two-dimensional covariate}

The sample considered here is made of $n=1,000$ independent realizations of a random vector $\left(X^{\prime}, Y\right)^{\prime}$, where $X=\left(X_{1}, X_{2}\right)^{\prime}$ is uniformly distributed on the square $(-2,2)^{2}$ and where $Y$ is obtained by adding to $X_{1}^{2}+X_{2}^{2}$ an independent standard normal error term.

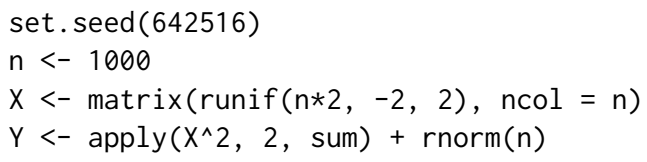

We test $N$ between 40 and 90 by steps of 10 . We change the values of $B$ and tildeB to reduce the computational burden, which is heavier for $d=2$ than for $d=1$. We keep the default values of all other arguments when running the function Quant i fQuantile.d2. Here, a warning message is printed informing us that testN was not well-chosen. We confirm it with the function plot with ise argument set to TRUE. 


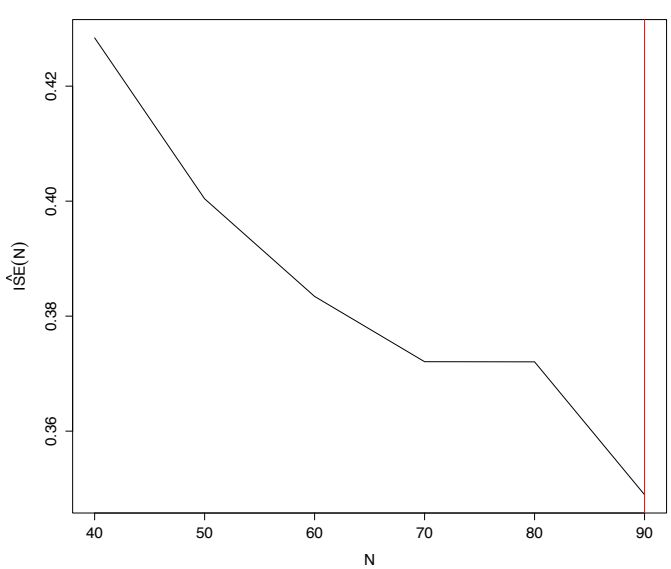

(a)

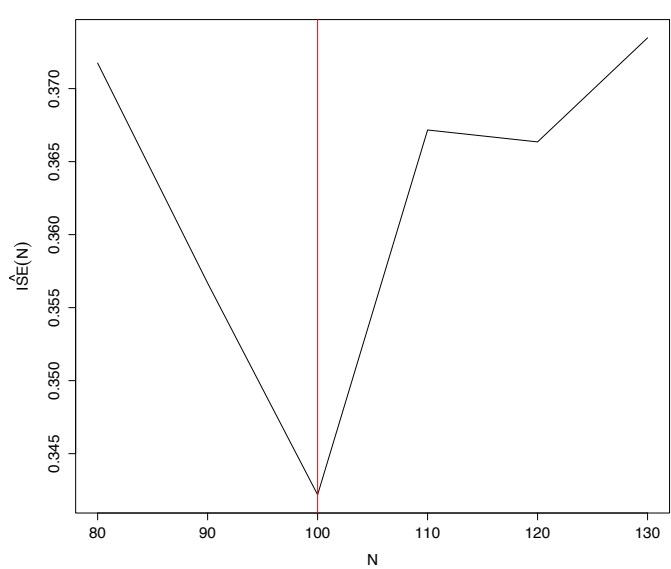

(b)

Figure 3: For the sample considered in Example 2, this figure plots $N \mapsto \widehat{\operatorname{ISE}}^{-}(N)$ (a) for $N \in$ $\{40,50,60,70,80\}$ and $(b)$ for $N \in\{80,90, \ldots, 120,130\}$.

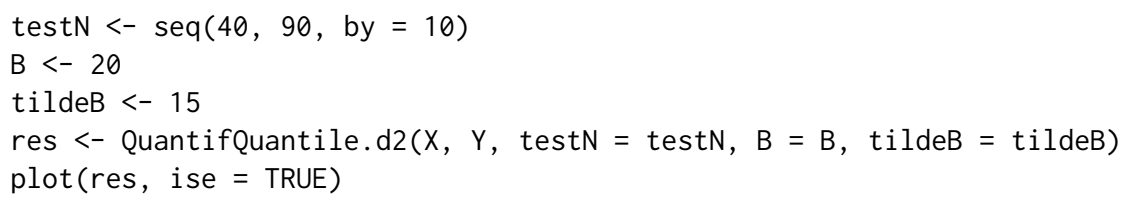

Figure 3a provides the resulting graph. The parameter testN was not well chosen since hat ISEmean_N becomes smaller and smaller as N_opt increases. We then adapt the choice of testN accordingly and rerun the procedure, which identifies an optimal $N$-value equal to 100; see Figure 3b.

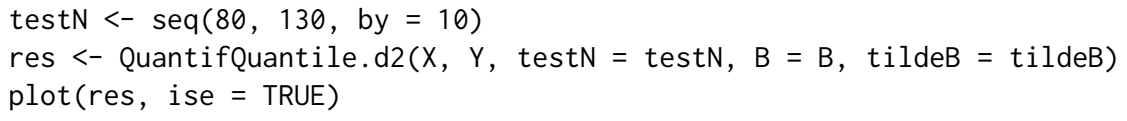

We then plot the corresponding estimated conditional quantile surfaces in Figure 4.

col.plot <- c("black", "red", "orange", "green", "orange", "red")

plot (res, col.plot $=$ col.plot, $\left.x l a b=" X_{-} 1 ", \quad y l a b=" X \_2 ", \quad z l a b=" Y "\right)$

\section{Example 3: Real data study and comparison with some competitors}

This example aims at illustrating the proposed estimated reference curves on a real data set and at comparing them with some competitors. In this example, the ncores parameter of Quanti fQuantile function was set to the number of cores detected by $\mathrm{R}$ minus 1 . The data used here, that are included in the QuantifQuantile package, involves several variables related to employment, housing and environment associated with $n=542$ towns/villages in Gironde, France. For the present illustration, we restrict to the regressions $R_{1}$ and $R_{2}$ involving $(X, Y)=$ (percentage of owners living in their primary residence, percentage of buildings area) and $(X, Y)=$ (percentage of middle-range employees, population density), respectively. In both cases, $n=542$ observations are available and we are interested in the estimation of reference curves for $\alpha=0.05,0.25,0.50,0.75$ and 0.95 . For both $R_{1}$ and $R_{2}$, we tested the number $N$ of quantizers to be used between 5 and 15 by step of 1 , using the methodology described in Example 1.

set.seed(644925)

data (gironde)

$X<-$ gironde[[2]]\$owners

$Y<-$ gironde[[4]]\$building

testN <- seq $(5,15$, by $=1)$

res <- QuantifQuantile $(X, Y$, testN $=\operatorname{testN}$, same_N $=F$, ncores $=\operatorname{detectCores}()-1)$

col.plot <- c("grey", "red", "orange", "green", "orange", "red")

plot $($ res, col.plot $=$ col.plot, $x l a b=" X ", y l a b=" Y ")$ 


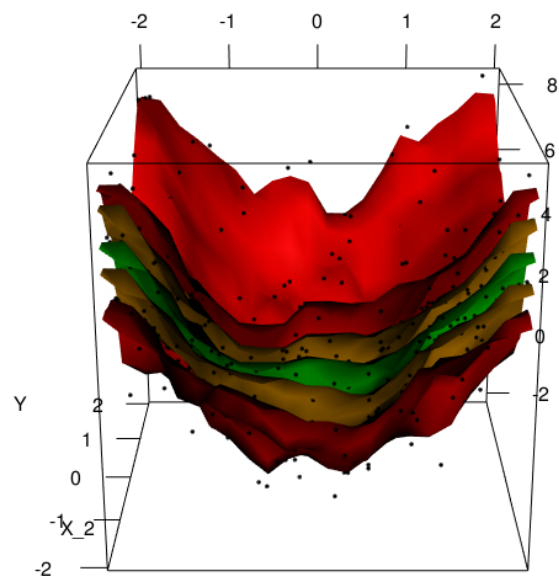

X_1

(a)

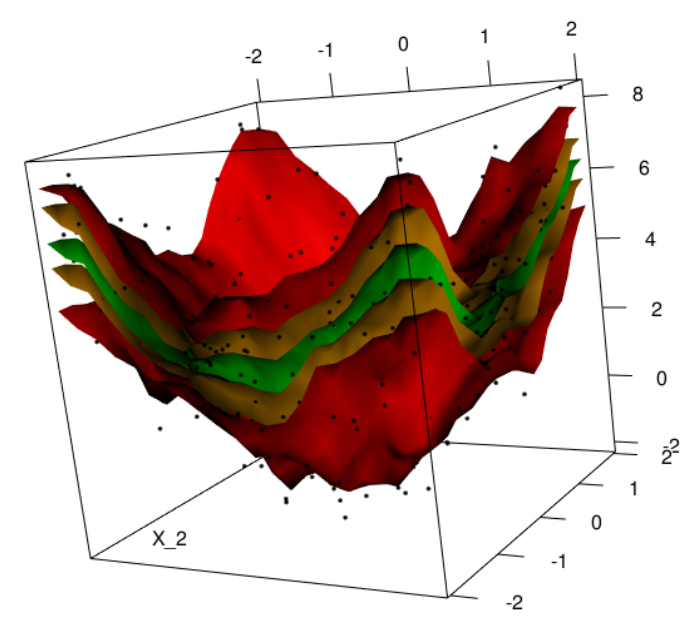

X_1

(b)

Figure 4: For the sample considered in Example 2, this figure plots (with two different views) the estimated conditional quantile surfaces obtained with the plot function for $\alpha=0.05,0.25,0.50,0.75$ and 0.95 .

The same exercise is repeated with $(X, Y)=$ (percentage of middle-range employees, population density). For each $\alpha$-value considered, we obtained $\hat{N}_{\alpha ; \text { opt }}^{-}=13$ for $R_{1}$ and $\hat{N}_{\alpha \text {;opt }}^{-}=7$ for $R_{2}$. The corresponding quantization-based reference curves are plotted in Figures 5 a and $5 c$, respectively. For the sake of comparison, spline-based curves are provided in Figures $5 \mathrm{~b}$ and $5 \mathrm{~d}$. These were obtained from the function rqss in the package quantreg. Since the parameter $\lambda$ involved, that governs the trade-off between fidelity and smoothness, is not automatically selected by rqss, we selected it through AIC (via the AIC function), separately for each order $\alpha$.

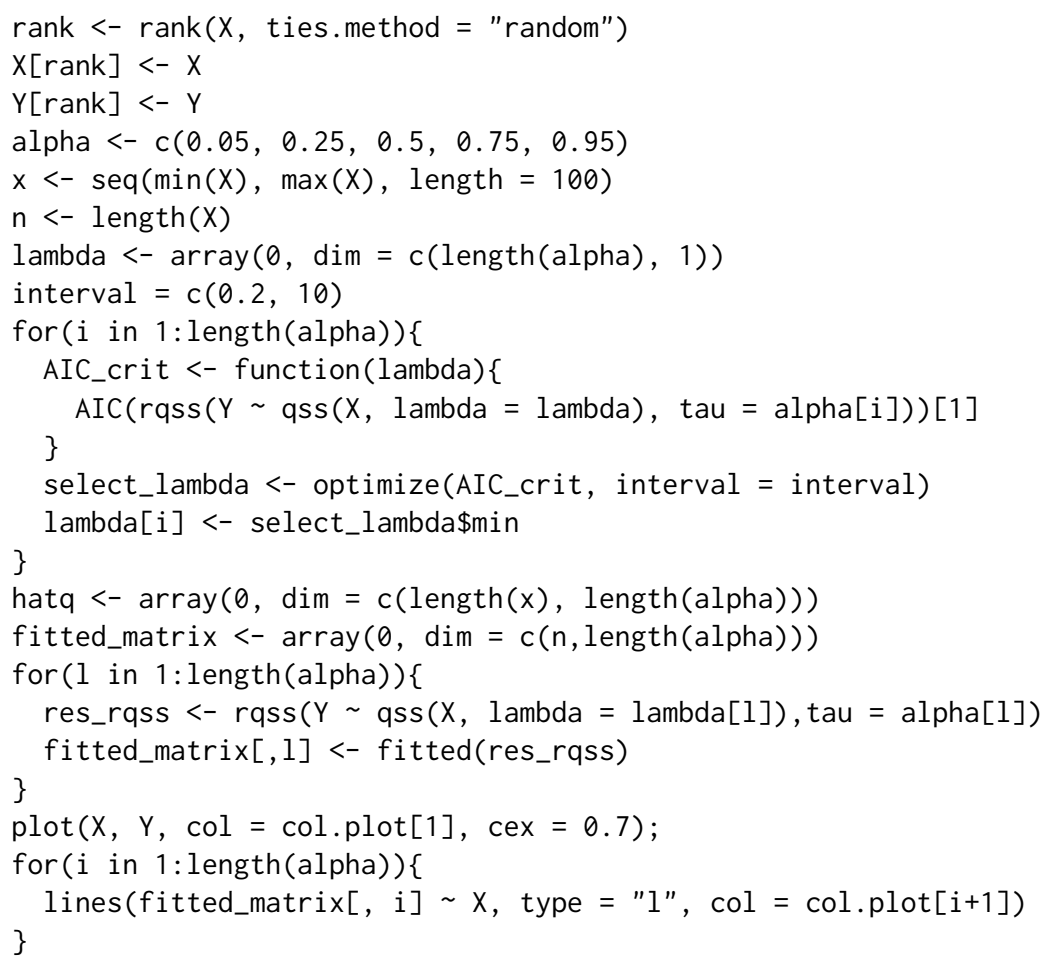

The same exercise is repeated for $R_{2}$, but with $\lambda$ tested between 0.5 and 15 . Since they are piecewise linear, the resulting spline-based reference curves are less smooth than the one based on quantization. Arguably, the latter better adapt to the samples even though they are sometimes quite wiggly.

Of course, the computational burden is also an important issue. Therefore, Table 1 gathers, for each method and each regression problem, the average and standard deviation of the computing times 


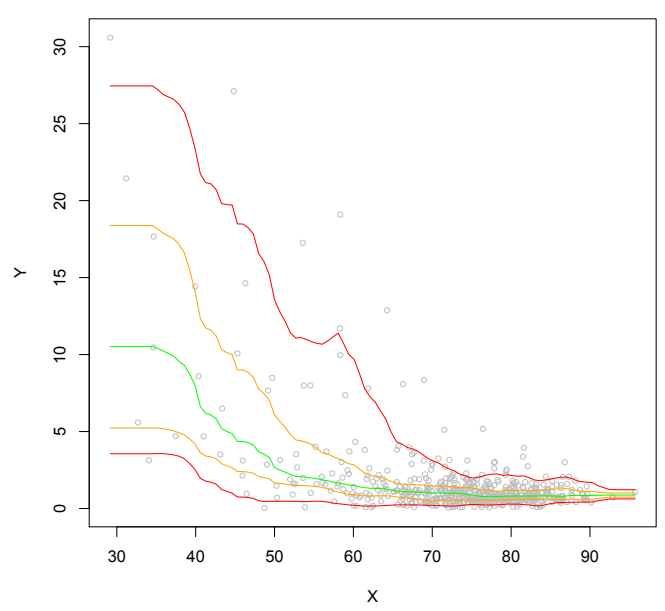

(a)

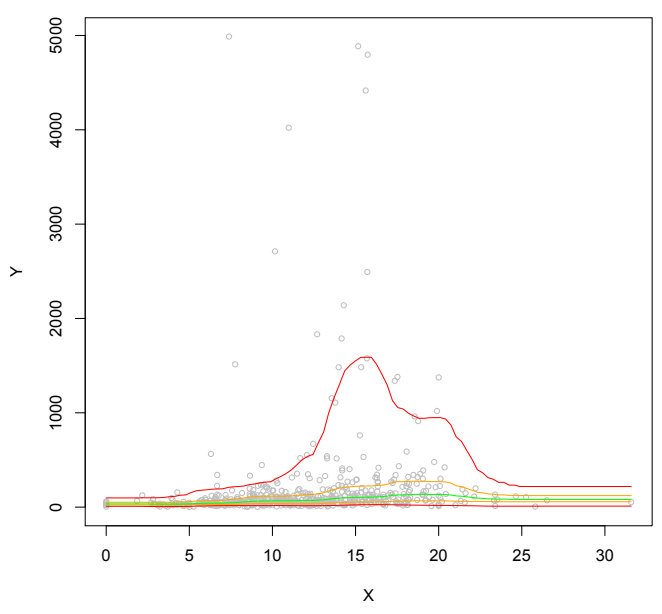

(c)

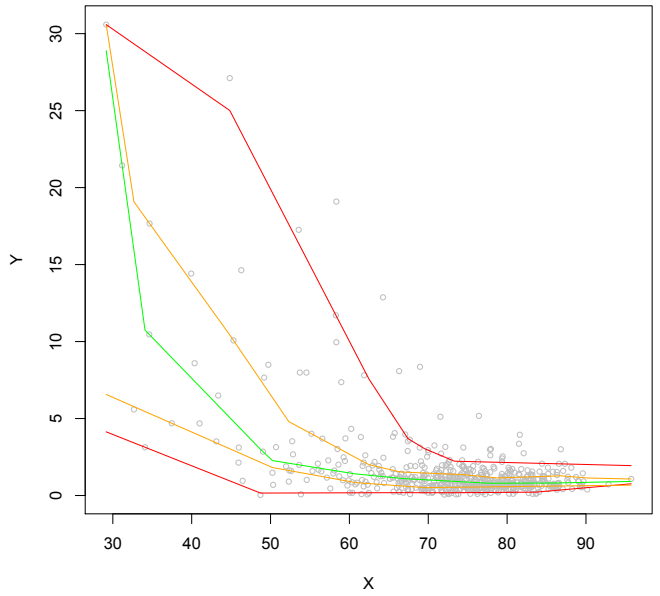

(b)

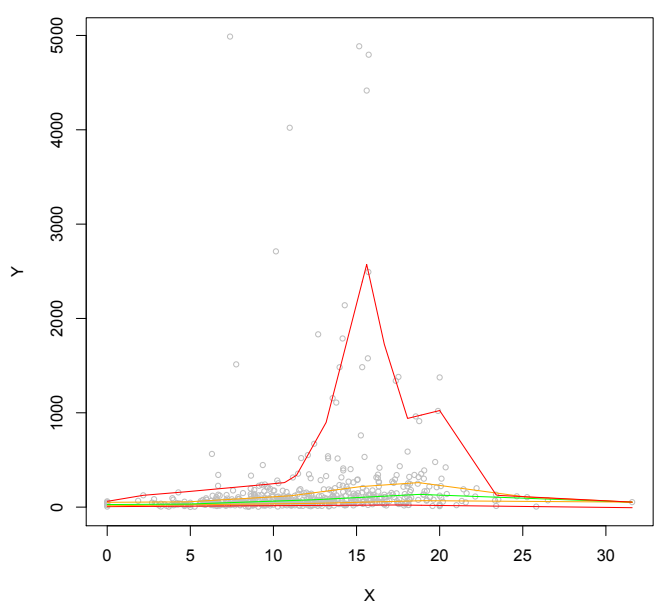

(d)

Figure 5: Estimated conditional quantile curves obtained with Quanti fQuantile (left) and rqss (right), for regression $R_{1}$ (top) and regression $R_{2}$ (bottom). In each case, the quantile orders considered are $\alpha=0.05,0.25,0.50,0.75$ and 0.95 .

\begin{tabular}{ccc}
\hline & QuantifQuantile & rqss \\
\hline$R_{1}$ & $2.83(0.117)$ & $4.39(0.119)$ \\
$R_{2}$ & $2.47(0.085)$ & $4.08(0.115)$ \\
\hline
\end{tabular}

Table 1: Averages of the computing times (in seconds) to obtain 50 times the conditional quantile curves in Example 3 for Quant i fQuantile and rqss, respectively; standard deviations are reported in parentheses.

in a collection of 50 runs (these 50 runs were considered to make results more reliable). In each case, our method is faster than its spline-based competitor.

\section{Example 4: Real data with three-dimensional covariate}

To treat an example with $d>2$, we reconsider the data set in Example 3, this time with the response $Y=$ population density and the three covariates $X_{1}=$ percentage of farmers, $X_{2}=$ percentage of unemployed workers, and $X_{3}=$ percentage of managers. In this setup, no graphical output is 
available. We therefore restrict to a finite collection of $x$-values where conditional quantiles are to be estimated. Denoting by $M_{j}$ and $\bar{X}_{j}, j=1,2,3$, the maximal value and the average of $X_{i j}$, $i=1, \ldots, n=542$, respectively, we consider the following eight values of $x$ :

$$
\begin{aligned}
& x_{1}=\left(\begin{array}{c}
\bar{X}_{1} \\
\bar{X}_{2} \\
\bar{X}_{3}
\end{array}\right), x_{2}=\left(\begin{array}{c}
\frac{1}{2}\left(\bar{X}_{1}+M_{1}\right) \\
\bar{X}_{2} \\
\bar{X}_{3}
\end{array}\right), x_{3}=\left(\begin{array}{c}
\bar{X}_{1} \\
\frac{1}{2}\left(\bar{X}_{2}+M_{2}\right) \\
\bar{X}_{3}
\end{array}\right), x_{4}=\left(\begin{array}{c}
\bar{X}_{1} \\
\bar{X}_{2} \\
\frac{1}{2}\left(\bar{X}_{3}+M_{3}\right)
\end{array}\right), \\
& x_{5}=\left(\begin{array}{c}
\frac{1}{2}\left(\bar{X}_{1}+M_{1}\right) \\
\frac{1}{2}\left(\bar{X}_{2}+M_{2}\right) \\
\bar{X}_{3}
\end{array}\right), x_{6}=\left(\begin{array}{c}
\frac{1}{2}\left(\bar{X}_{1}+M_{1}\right) \\
\bar{X}_{2} \\
\frac{1}{2}\left(\bar{X}_{3}+M_{3}\right)
\end{array}\right), x_{7}=\left(\begin{array}{c}
\frac{1}{2}\left(\bar{X}_{2}+M_{2}\right) \\
\frac{1}{2}\left(\bar{X}_{3}+M_{1}\right)
\end{array}\right), x_{8}=\left(\begin{array}{c}
\frac{1}{2}\left(\bar{X}_{2}+M_{2}\right) \\
\frac{1}{2}\left(\bar{X}_{3}+M_{3}\right)
\end{array}\right) .
\end{aligned}
$$

The function QuantifQuantile.d is then evaluated for the response and covariates indicated above, and with the arguments alpha $=(0.25,0.5,0.75)^{\prime}$, testN $=(5,6,7,8,9,10)^{\prime}$, $\mathrm{x}$ being the $3 \times 8$ matrix whose columns are the vectors $x_{1}, x_{2}, \ldots, x_{8}$ just defined and ncores being the number of cores detected by $\mathrm{R}$ minus 1 .

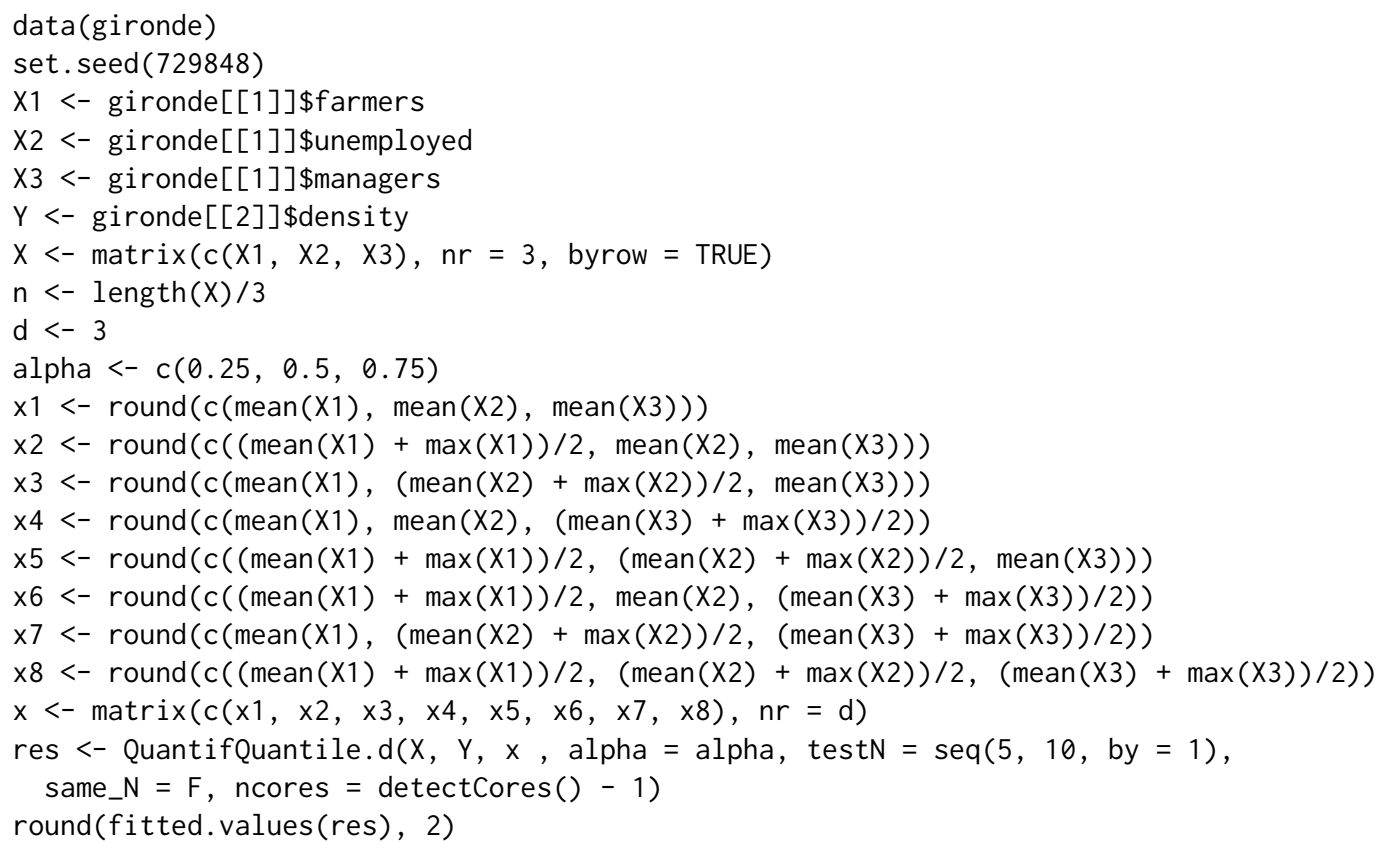

This provided $\hat{N}_{\alpha ; \text { opt }}^{-}=8,7$ and 7 , for $\alpha=0.25,0.50$ and 0.75 , respectively. The total computation time is 6.86 seconds. The fitted.values function then allowed to return the following matrix hatq_opt of estimated conditional quantiles :

$$
[, 1] \quad[, 2] \quad[, 3] \quad[, 4] \quad[, 5] \quad[, 6] \quad[, 7] \quad[, 8]
$$

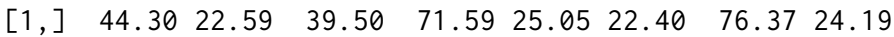

$\left[\begin{array}{llllllllll}{[2,]} & 80.07 & 32.31 & 81.24 & 161.85 & 35.01 & 31.92 & 145.29 & 38.18\end{array}\right.$

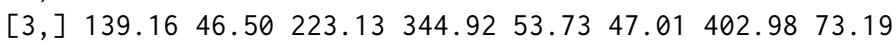

This collection of (estimated) conditional quartiles allows to appreciate the impact of a marginal perturbation of the covariates on $Y^{\prime}$ 's conditional median (location) or interquartile range (scale). For instance, the results suggest that $Y^{\prime}$ 's conditional median decreases with $X_{1}$, is stable with $X_{2}$, and increases with $X_{3}$, whereas its conditional interquartile range decreases with $X_{1}$ but increases much with $X_{2}$ and with $X_{3}$. The eight $x$-values considered further allow to look at the joint impact of two or three covariates on $Y^{\prime}$ 's conditional location and scale. Of course, other shifts in the covariates (and other orders $\alpha$ ) should further be considered to fully appreciate the dependence of $Y$ on $X$.

\section{Conclusion}

In this paper, we described the package QuantifQuantile that allows to implement the quantizationbased quantile regression method introduced in Charlier et al. $(2015 a, b)$. The package is simple to use, as the function QuantifQuantile and its multivariate versions essentially only require providing the covariate and response as arguments. Since the choice of the tuning parameter $N$ is crucial, a warning 
message is printed if it is not well-chosen and the function plot can also be used as guide to change adequately the value of the parameter testN in the various functions. Moreover, a graphical illustration is directly provided by the same function plot when the dimension of the covariate is smaller than or equal to 2. Finally, this package also contains a function that provides optimal quantization grids, which might be useful in other contexts, too.

Finally, we stress that quantization-based estimators, like most nonparametric smoothing procedures, are likely to perform poorly in high-dimensional situations due to the curse of dimensionality. For large $d$, it is therefore unclear how to assess whether a given covariate has a significant impact on the response variable. For small $d$, however, it is always possible, in the absence of a formal testing procedure, to resort to visual inspection. In the simplest case of a single covariate $(d=1)$, this would lead to looking whether or not fitted curves approximately are horizontal lines. This can be extended to the case $d=2$.

\section{Acknowledgments}

The authors are grateful to the Editor and two anonymous referees for their careful reading and insightful comments that led to a significant improvement of the original manuscript. The first author's research is supported by a Bourse FRIA of the Fonds National de la Recherche Scientifique, Communauté française de Belgique. The second author's research is supported by an A.R.C. contract from the Communauté Française de Belgique and by the IAP research network grant P7/06 of the Belgian government (Belgian Science Policy).

\section{Appendix}

\section{Illustration of choice.grid}

We here put to work the function choice.grid in the univariate and bivariate cases. This function provides the "optimal" grid generated by the stochastic gradient algorithm described earlier. As above mentioned, quantization was extensively used in many other fields as numerical integration, cluster analysis, numerical probability or finance (Pagès, 1998; Pagès et al., 2004). Therefore, this function can be of interest outside the regression setup considered here.

We start with the univariate case and generate a random sample of size $n=500$ from the uniform distribution over $(-2,2)$. With $\mathrm{N}=15$ and $\mathrm{ng}=1$, this function provides a single initial grid (obtained by sampling without replacement among the uniform sample) and the corresponding optimal grid returned by the algorithm. Figure 6 represents the observations (in grey), the initial grid (in red), and the optimal grid (in green). The same exercise is repeated with sample size $n=5,000$, and the results are also given in Figure 6.

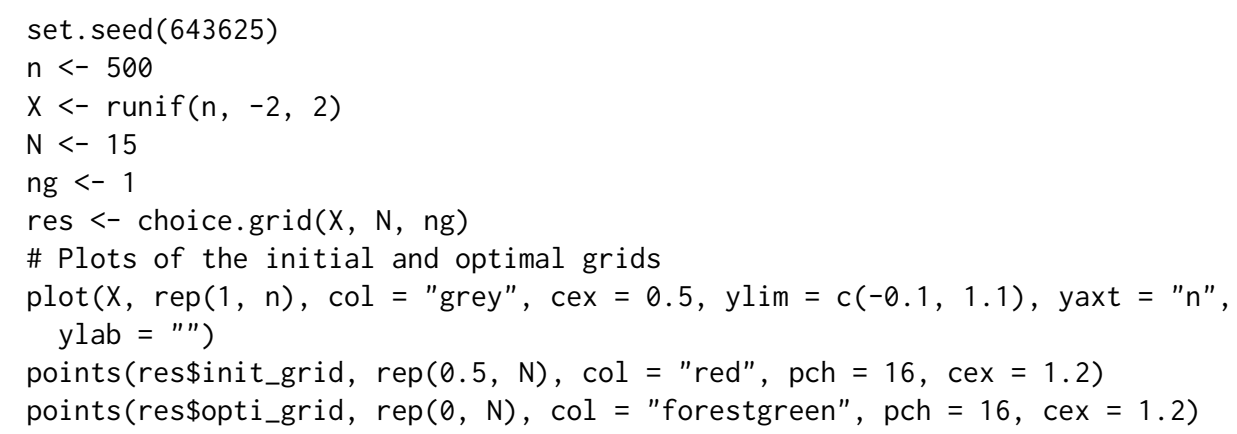

Since the parent distribution is uniform over $(-2,2)$, the population optimal grid is the equispaced grid on that interval (Pagès, 1998). For both sample sizes considered, the optimal grid provided by the choice.grid function is much closer to the population optimal grid than the initial one. Recalling that the stochastic gradient algorithm in choice.grid performs as many iterations as observations in the original sample, it is not surprising that the optimal grid associated with the sample of size 5, 000 better approximates the population optimal grid than the optimal grid associated with the sample of size 500 .

Finally, we turn to the bivariate case and generate two random samples of size $n=2,000$ and size $n=20,000$ from the uniform distribution over the square $(-2,2)^{2}$. The function choice.grid was applied to these samples with $\mathrm{N}=30$ and $\mathrm{ng}=1$. The resulting couple of initial and optimal grids are plotted in Figure 7. As in the univariate case, we observe an improvement when going from the initial grids to the corresponding optimal grids provided by the function choice.grid (here as well, the 


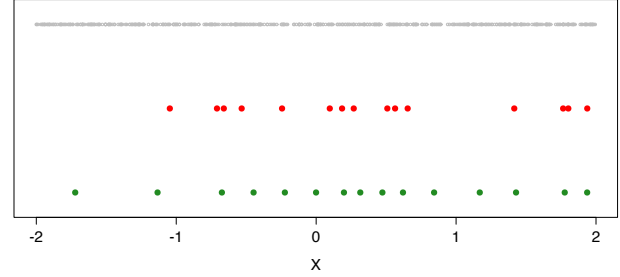

(a)

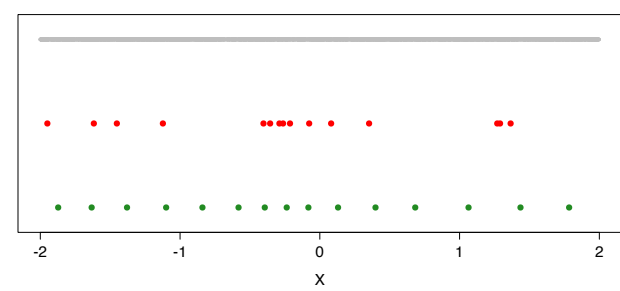

(b)

Figure 6: For $n=500$ (left) and $n=5,000$ (right), a random sample of size $n$ from the uniform distribution over $(-2,2)$ (in grey), an initial grid of size 15 obtained by sampling without replacement among these $n$ observations (in red), and the "optimal" grid returned by choice.grid (in green).

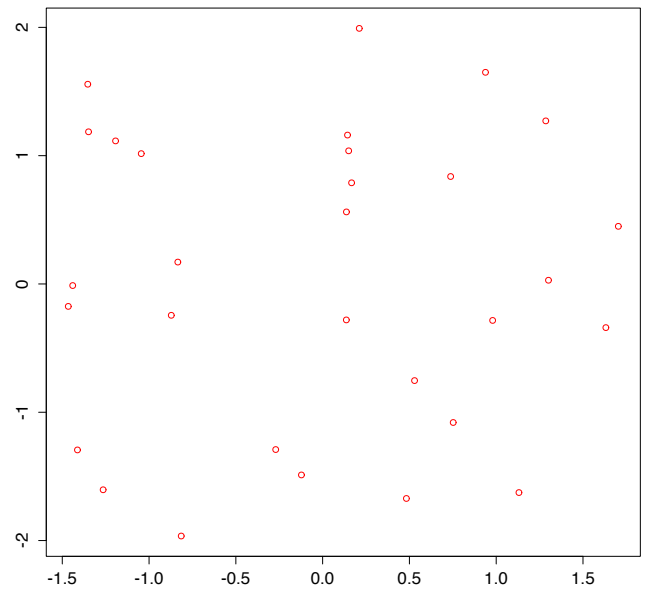

(a)

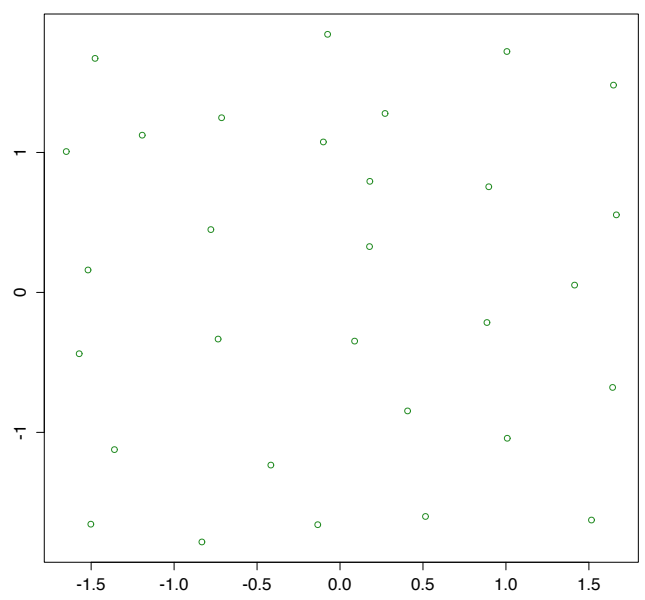

(c)

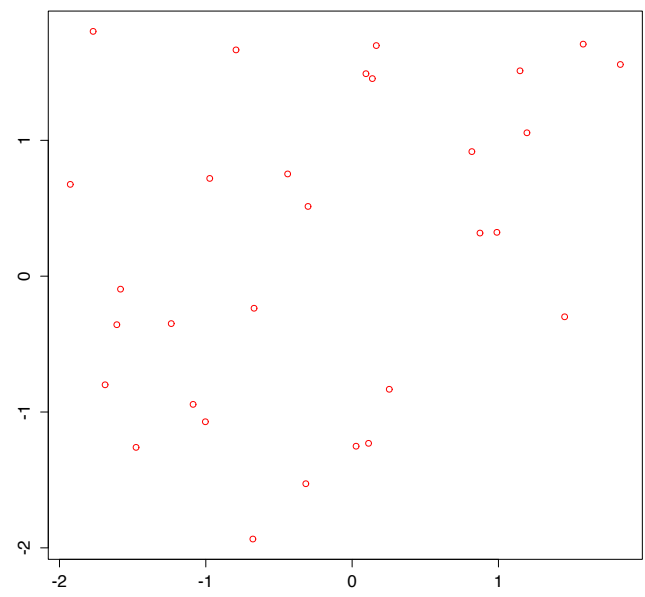

(b)

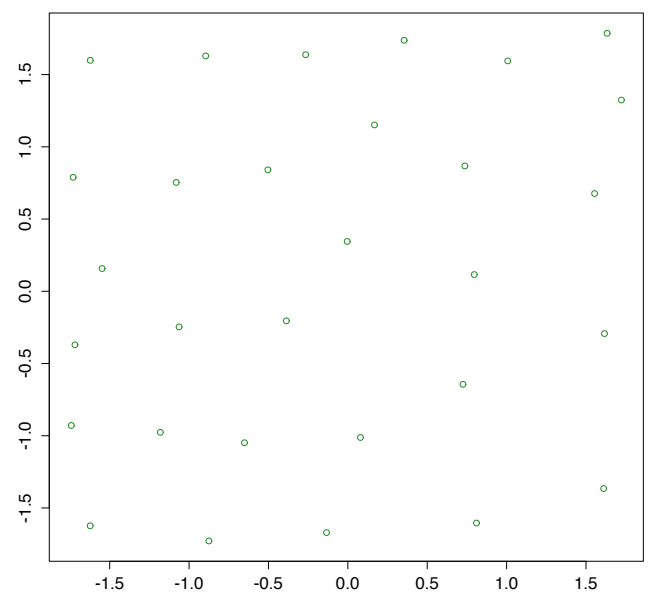

(d)

Figure 7: For $n=2,000$ (left) and $n=20,000$ (right), an initial grid of size 15 obtained by sampling without replacement among a random sample of size $n$ from the uniform distribution over $(-2,2)$ (top), and the corresponding optimal grid returned by choice.grid (bottom).

population optimal grid should be uniformly spread over the support of the underlying distribution). Also, it is still the case that the resulting optimal grid is better when based on a larger sample size $n$. 


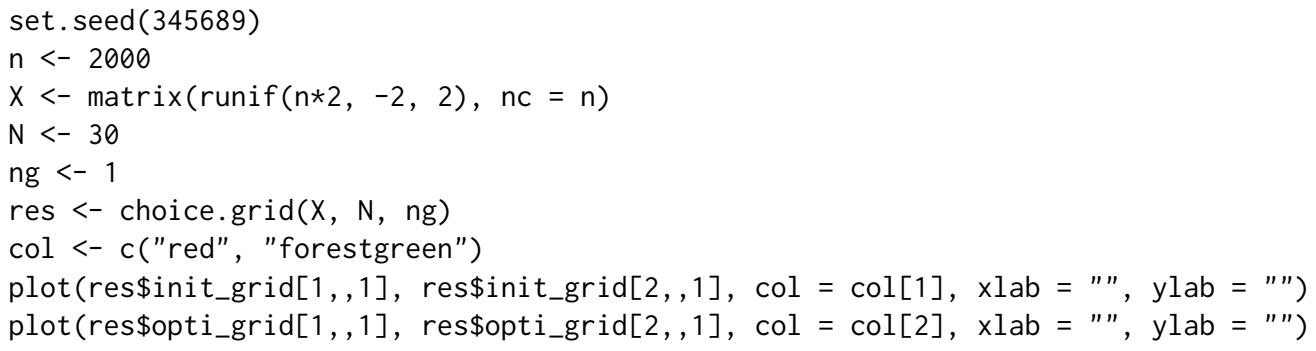

\section{Bibliography}

D. Adler, D. Murdoch, and others. rgl: 3D Visualization Device System (OpenGL), 2015. URL https: //CRAN. R-project. org/package=rgl. R package version 0.95.1367. [p69]

P. K. Bhattacharya and A. K. Gangopadhyay. Kernel and nearest-neighbor estimation of a conditional quantile. The Annals of Statistics, 18(3):1400-1415, 1990. [p65]

I. Charlier, D. Paindaveine, and J. Saracco. Conditional quantile estimation through optimal quantization. Journal Statistical Planning and Inference, 156:14-30, 2015a. [p65, 66, 67, 76]

I. Charlier, D. Paindaveine, and J. Saracco. Conditional quantile estimation based on optimal quantization: from theory to practice. Computational Statistics $\mathcal{E}$ Data Analysis, 91:20-39, 2015b. [p65, 66, 67, $68,76]$

I. Charlier, D. Paindaveine, and J. Saracco. QuantifQuantile: Estimation of Conditional Quantiles using Optimal Quantization, 2015c. URL https://CRAN.R-project.org/package=QuantifQuantile. R package version 2.0. [p65]

J. Fan, T.-C. Hu, and Y. Truong. Robust nonparametric function estimation. Scandinavian Journal of Statistics, 21(4):433-446, 1994. [p65]

A. Gannoun, S. Girard, C. Guinot, and J. Saracco. Reference curves based on non-parametric quantile regression. Statistics in Medicine, 21(4):3119-3135, 2002. [p65]

S. Graf and H. Luschgy. Foundations of Quantization for Probability Distributions, volume 1730 of Lecture Notes in Mathematics. Springer-Verlag, Berlin, 2000. [p66]

R. Koenker. quantreg: Quantile Regression, 2015. URL https://CRAN.R-project.org/package= quantreg. R package version 5.19. [p65]

R. Koenker and G. Bassett, Jr. Regression quantiles. Econometrica, 46(1):33-50, 1978. [p65]

R. Koenker and I. Mizera. Penalized triograms: Total variation regularization for bivariate smoothing. Journal of the Royal Statistical Society, Series B (Statistical Methodology), 66(1):145-163, 2004. [p65]

R. Koenker, P. Ng, and S. Portnoy. Quantile smoothing splines. Biometrika, 81(4):673-680, 1994. [p65, 68]

V. M. R. Muggeo. quantregGrowth: Growth Charts via Regression Quantiles, 2015. URL https: //CRAN. Rproject. org/package=quantregGrowth. $\mathrm{R}$ package version 0.3-1. [p65]

V. M. R. Muggeo, M. Sciandra, A. Tomasello, and S. Calvo. Estimating growth charts via nonparametric quantile regression: A practical framework with application in ecology. Environmental and Ecological Statistics, 20(4):519-531, 2013. [p65]

G. Pagès. A space quantization method for numerical integration. Journal of Computational and Applied Mathematics, 89(1):1-38, 1998. [p66,77]

G. Pagès, H. Pham, and J. Printems. Optimal quantization methods and applications to numerical problems in finance. In Handbook of Computational and Numerical Methods in Finance, pages 253-297. Birkhäuser Boston, Boston, MA, 2004. [p66, 77]

K. Yu and M. C. Jones. Local linear quantile regression. Journal of the American Statistical Association, 93 (441):228-237, 1998. [p65, 68]

K. Yu, Z. Lu, and J. Stander. Quantile regression: Applications and current research areas. Journal of the Royal Statistical Society, Series D (The Statistician), 52(3):331-350, 2003. [p65] 
Isabelle Charlier

Université Libre de Bruxelles

Département de Mathématique and ECARES

Campus Plaine, Boulevard du Triomphe, CP210

1050 Brussels

Belgium

and

Université de Bordeaux

IMB, UMR 5251

33400 Talence

France

and

Inria Bordeaux Sud-Ouest

200 Avenue Vieille Tour

33400 Talence

France

ischarli@ulb.ac.be

Davy Paindaveine

Université Libre de Bruxelles

ECARES and Département de Mathématique

Avenue F.D. Roosevelt, 50, ECARES - CP114/04

1050 Brussels

Belgium

dpaindav@ulb.ac.be

Jérôme Saracco

Université de Bordeaux

IMB, UMR 5251

33400 Talence

France

and

Inria Bordeaux Sud-Ouest

200 Avenue Vieille Tour

33400 Talence

France

Jerome. Saracco@math.u-bordeaux1.fr 